\title{
A Structured Approach to Care for Persons with Multiple Sclerosis: Need of the Hour
}

\section{Vasundharaa S Nair ${ }^{1}$, Priya Treesa Thomas ${ }^{2 *}$, Netravathi $\mathrm{M}^{3}$ and Bhuvaneshwari $^{1}$}

${ }^{1}$ PhD Scholar, Department of Psychiatric Social Work, National Institute of Mental

Health and Neurosciences, Bengaluru, India

${ }^{2}$ Associate Professor, Department of Psychiatric Social Work, National Institute of

Mental Health and Neurosciences, Bengaluru, India

${ }^{3}$ Additional Professor, Department of Neurology, National Institute of Mental

Health and Neurosciences, Bengaluru, India

*Corresponding Author: Priya Treesa Thomas, Associate Professor, Department

of Psychiatric Social Work, National Institute of Mental Health and Neurosciences,

Bengaluru, India.
Received: March 01, 2021

Published: March 24, 0221

(C) All rights are reserved by Priya Treesa

Thomas., et al.

\begin{abstract}
Introduction: Multiple sclerosis (MS) is an auto-immune disease which involves an abnormal response of the body's immune system directed against the central nervous system (CNS) - brain, spinal cord and optic nerves being involved. Suicide is defined as the act of killing yourself, most often as a result of chronic conditions.

Objective: The study aims to gain insight into the presence of suicidal ideations among Persons with Multiple Sclerosis and the effect of the same on their Quality of Life.

Methodology: To achieve the purpose of the study, the patients presenting to the Multiple Sclerosis clinic of Department of Neurology were assessed using the Multiple Sclerosis Quality of Life Scale (MS-QOL), Expanded Disability Status Scale (EDSS) and the Scale for Suicide Ideation (SSI). Based on the assessment individualized interventions were carried out focusing on their perception, precautions and the necessary preventive strategies to be taken.

Results: The results have shown the presence of suicidal ideations among the group, with the difficulties faced by the population thereby indicating the need for a structured intervention.

Conclusion: In the growing context of patients with Multiple Sclerosis and the associated disability and the chronicity, it becomes imperative, to focus on the presence, effect, perception and the protecting factors to establish the preventive strategies which shall thereby help in understanding of the method of suicide prevention and ensuring better quality of life helping in sensitivity and positivity towards mental health.
\end{abstract}

Keywords: Multiple Sclerosis; Suicide; Psychosocial Care; Multidisciplinary Approach to Care

\section{Introduction}

Multiple Sclerosis (MS) is an auto-immune condition that involves an abnormal response of the body's immune system which is directed against the central nervous system- being involved in the brain, spinal cord, and optic nerves (National Multiple Sclerosis Society, 2012). The association between Multiple Sclerosis and psy- 
chosocial stress factors is well-established with concerns ranging from mood changes to Generalized anxiety and distress [1]. Wish to die as a phenomenon is considered common among chronic neurological conditions like Multiple Sclerosis [2] making suicide- "the act of killing yourself" a factor that highlights the need for action. The recent COVID 19 pandemic has played havoc with the chronic disease management, thus affecting the access to necessary medicines and follow up.

\section{Materials and Methods}

A cross sectional descriptive study was conducted among thirty participants (both male and female). All participants had the diagnosis of Multiple Sclerosis and were seeking treatment in a multidisciplinary national tertiary referral care center for neuropsychiatric diseases in South India. The study was conducted as part of routine service delivery to gain insight into the presence of suicide risk. Participants were interviewed from (April 2020 to June 2020). A semi-structured schedule and standardized scales Multiple Sclerosis-Quality of Life (MS-QoL; Preedy and Watson [3]), Expanded Disability Status Score (EDSS; Kurtz, [4]), and Scale for Suicidal Ideation (SSI; Beck., et al. [5]) were administered. Most of the participants had the diagnosis of Remitting Relapsing type and Secondary Progressive Type (77\%; 23\%). Socio-demographic details showed the mean age as $38.4+17.92$ with a gender ratio of 7:3 (Female: Male). 70\% belonged to the Middle Socio-Economic Status. The assessments were carried out through interviews conducted in person in the clinic (13 participants) as well as through the routine follow up calls from the clinic (17 participants) during the COVID 19 pandemic. The data was analyzed using descriptive and inferential statistics and the responses to the open ended questions were thematically analyzed.

\section{Results}

The overall Quality of Life was reported to be average with the mean score of $6.4+$ /- 3.06 with predominant concerns across general health, health distress, cognitive functioning, sexual health, and life as a whole. Though $60 \%$ were on medications, $70 \%$ percent of the respondents had a relapse in the last 6 months with the disability mean score as $4.3+/-1.9$ (stating fully ambulatory condition with the ability to work a full day with the provision of minimal assistance) on the EDSS scale. On the suicide ideation scale, $40 \%$ reported attempting suicide, and $63 \%$ with active suicidal ideation. The protective factors were found as the family structure and the religious beliefs of the person with the presence of suicidal ideation in $63 \%$ of the population.
Major themes being that emerged from the qualitative interviews were as follows.

Feeling of distress: Participants reported being distressed about the episodes of neurological illness and the impact of it in her life.

"I was suicidal a year ago, when I had my first attack, I remember my sister taking me around places to understand my difficulty and post getting the diagnosis from NIMHANS, we came back home. When I came back, the man I trusted and loved let go of our years bond because of my illness, I was extremely hurt, I hated myself and cursed God for giving me the condition and even thought of committing". (Ms. J, 27 years, EDSS - 6, duration of 6 years).

Increasing difficulty in living a regular life: The neurological problems associated with Multiple sclerosis brought about marked changes in the everyday life of the participants, in many cases resulting in drastically changes in lifestyle and limited choices.

"My life changed overnight. I was a high performing employee awaiting my promotion when I got my first attack. I left my job due to my difficulty and was at home. I then started preparing for civil services but then my condition worsened. Though I am on medicines, telling people about my conditions especially to people who are coming for matrimonial proposals, and then making them understand is so difficult and at times makes me very sad. But I am trying to make myself better. I have spoken to all of you and my family is very supportive, so I am fine now". (Ms. M, 26 years, EDSS4.5 , duration of 5 years).

Feeling of being a burden: Participants described the devastating effect of the neurological disability in their life. Being dependent on family members for everyday activities was emotionally draining, and each chore that needed to be supported brought this feeling to the forefront daily. Many participants felt this factor to be directly resulting in the thoughts about wishing to die, so that they are no longer a burden to their family.

"My life has changed completely. I have no wish to live. I feel ashamed that I have to depend on my sons for everything - even to go to the bathroom. I taught them and took them to the bathroom and now they have to do it, I can't even explain the level of shame I feel. I keep telling God to take my life such that my wife and sons have less burden. My sons are not even married, they have an entire life ahead of them, and here they are running around taking care of me. What has my life come to! I don't understand why I am 
dealing with this. Look at me, I can't even eat on my own- my body does not support me in any manner. I am just blessed that my wife and children have not given up on me. I would not even curse my enemies to have a life like me". (Mr. S, 36 years, EDSS- 8, duration of 10 years).

\section{Discussion}

The current study, conducted as part of routine service delivery in a multidisciplinary Multiple Sclerosis clinic, has attempted to explore the presence of suicidal ideations among the group.

The illness and associated disability have an effect on activities of daily living in the form of impaired functionality/loss of mobility. These increase the risk of having breathing problems and aspiration pneumonia. It is important to note that the body's natural immune response may also make the symptoms worse [6].

There was significant difficulty in accessing medicines, which has been aggravated by the ongoing COVID 19 pandemic. Drug default and the difficulty in procuring the medications could one of the strong elements that may increase the chances of a relapse and associated residual damage in a person with MS. This thereby shall have a direct correlation with the quality of life and well-being of a person and their family.

Multiple Sclerosis being a chronic inflammatory disease of the central nervous system has been found to have a gender ratio of 2.3-3.5:1 (woman: man) [7]. Increased time spent by the larger family results in increased role strain on the women resulting in she is taking care of herself and putting extra efforts to fulfill the duties and responsibilities of the house.

Individuals isolated in different places without access to a healthcare facility and/or a primary or secondary support system face difficulty in connecting to health care centers and healthcare professionals to cater to the physical and mental health needs. The influx of myths without the awareness of the necessary knowledge, attitudes, and practices could be a major hindrance towards the receiving care and thereby establishing the continuum of care.

To manage these concerns a structured intervention that is developed based on the chronic care model (Wagner., et al. [8], figure 1 ) is being suggested as a part of the multidisciplinary team involving:

- Establishment of a care structure: It is important to segment the care at the individual, family ,and community lev-

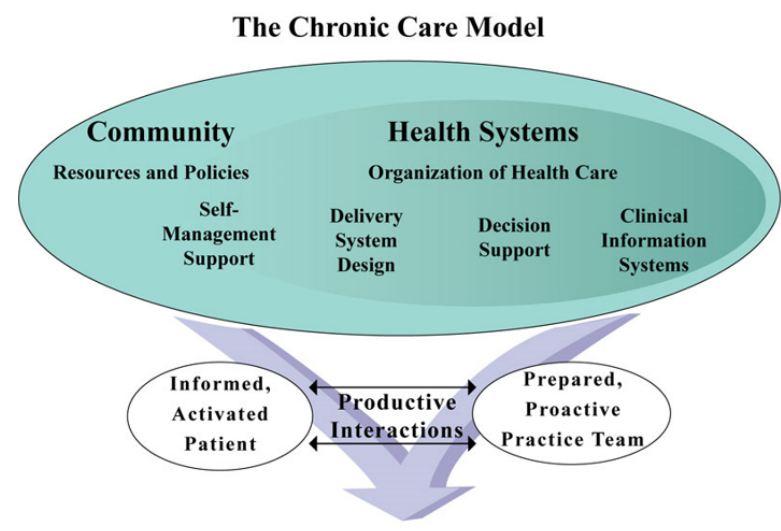

Improved Outcomes

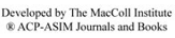

Figure 1: Wagner., et al. 2009.

els. Individual therapy, self-care management, family stress management, and community level resource utilization are the need of the hour.

- Adherence to and support for the medical regime: Disruption in their locality results in the non-availability of pharmacies to procure medicines making them susceptible to drug default. District Mental Health Hospitals and the associated pharmacy members could be liasoned with them to ensure the continuum of care.

- Setting of a boundary: Establishing the balance is one of the most crucial aspects of good functionality. Though the nature of work as "domestic work" increases the toll, learn to establish and divide the roles amongst all family members making it a joint effort towards family well-being.

- Taking care of one's general wellbeing over and above the illness: Ensuring sleep, food intake by being connected with loved ones, and enjoying one's work routine with elements of exercise and meditation shall help in a better quality of life.

- Apt use of technology: There is always a fine line between being aware and being overwhelmed with information. Persons with Multiple Sclerosis being a group more susceptible to internal and external forms of stress, they should be informed about where to seek the right information and help regarding their disease. 
- $\quad$ Reach out: Provision of helpline numbers, email addresses as well with the knowledge of the availability of the emergency services in times of increased severity of the condition shall reassure the patient and their caregivers that there are individuals that they could rely on always, thereby reducing their health-related anxiety and distress.

- Remember none of us are alone: Suffering is universal, and the disease, though not common, affects more people than expected. As much as the stressors create the distress, seeking help and reaching out to others through support groups and healthcare interactions will he helpful.

These interventions are though currently at the micro-level provided in the clinical setting need to be understood at a macro level based on the Chronic Care Model should be utilized.

\section{Limitations of the Study}

The study has several limitations such as a smaller sample size and the focus on the patients who seek care from a tertiary healthcare Centre.

\section{Conclusion}

COVID-19 pandemic has created more distress for patient with Multiple sclerosis creates a dual-stress on the Persons living with Multiple Sclerosis making it overwhelming for the person and taxing for the caregivers. There is a complex relationship between the disease and the presence of death wishes in this group, that needs to be carefully and sensitively explored in routine assessments. Ensuring the continuum of care from Bio-Psycho-Social model [9] of intervention implementation could help in ensuring the required medications thereby reducing the chances of relapse, shall make sure that the necessary psychological concerns are addressed through the necessary individual-level interventions and also shall help the family and other caregivers to both present and resolve the associated familial problems thereby establishing healthy homeostasis and better adaptation to the illness. This multidisciplinary approach shall help in ensuring the reduced disability-adjusted life years lost and ensuring a better quality of life.

\section{Bibliography}

1. National MS Society. "Emotional Changes". Retrieved from National MS Society (2012).

2. American Psychological Association. "Suicide". Retrieved from American Psychological Association (2013).
3. Preedy V R and Watson R R. "Handbook of disease burdens and quality of life measures". New York: Springer 6 (2010): 4265 .

4. Kurtzke JF. "Rating neurologic impairment in multiple sclerosis: an expanded disability status scale (EDSS)". Neurology 33.11 (1983): 1444-1452.

5. Beck A T., et al. "Assessment of suicidal intention: The Scale for Suicidal Ideation". Journal of Consulting and Clinical Psychology 47.2 (1976): 343-352.

6. Villines Z. "Multiple sclerosis (MS) and COVID-19: What to know". Medical News Today (2020).

7. Harbo H F., et al. "Sex and gender issues in multiple sclerosis". Therapeutic Advances in Neurological Disorders 6.4 (2013): 237-248.

8. Wagner EH., et al. "Evidence On The Chronic Care Model In The New Millennium. Improving Chronic Illness Care". MacColl Center for Health Care Innovation (2009).

9. Brofenbrenner U. "The ecology of human development: Experiments by nature and design". Harvard University Press (1979).

\section{Assets from publication with us}

- Prompt Acknowledgement after receiving the article

- Thorough Double blinded peer review

- Rapid Publication

- Issue of Publication Certificate

- High visibility of your Published work

Website: www.actascientific.com/

Submit Article: www.actascientific.com/submission.php Email us: editor@actascientific.com

Contact us: +919182824667 